\title{
Constipation due to a stroke complicated with pseudo-obstruction (Ogilvie's Syndrome)
}

\author{
Mariusz Madalinski \\ Royal Stoke University Hospital, Stoke-on-Trent, United Kingdom, \\ Northern Care Alliance NHS Group, Royal Oldham Hospital, United Kingdom
}

Key words: botulinum toxin, bowel pseudo-obstruction, stroke, anal sphincter

(Neurol Neurochir Pol 2021; 55 (2): 230-232)

\section{To the Editors:}

Constipation often occurs after a stroke, with an incidence of 29-79\% [1]. Although dysfunction of the brain-gut axis in stroke is recognised as the main cause of changes in bowel movement, several other factors can also contribute to constipation. Examples include reduced physical mobility and reduced fluid and/or fibre intake, especially in patients with associated dysphagia. Medication can affect bowel movement function, and there are also psychological aspects: depending on others to be able to use a toilet can lead to constipation too [1].

Constipation is probably also an independent risk of ischaemic stroke and coronary heart disease events (CHDE). This was demonstrated in a retrospective cohort study of over 3 million US veterans with a glomerular filtration rate (eGFR) $\geq 60 \mathrm{~mL} / \mathrm{min} / 1.73 \mathrm{~m}^{2}$. The study found that constipation was linked to a higher risk of the incidence of ischaemic stroke (19\%) and of CHDE (11\%) [2]. A prospective study of 45,112 Japanese men and women with a 13.3-year follow-up showed that a lower defecation frequency is associated with a higher risk of overall cardiovascular disease mortality and all forms of stroke mortality [3].

Constipation is accompanied by intestinal dysbiosis; chronic inflammation partly due to bacterial endotoxins or altered gut metabolites may serve as a potential explanation for the observed associations [4]. It remains unclear whether the microbiota have an impact on the outcome of acute brain injury, but some studies have shown that alterations in the intestinal flora can reduce ischaemic brain injury in a rat model - an effect which can be transmissible by faecal transplants [5].
Nowak et al. [6] reported constipation due to a stroke complicated with pseudo-obstruction. This condition can be categorised as either acute or chronic in nature [7]. Chronic idiopathic intestinal pseudo-obstruction is clinically divided into two types: small intestinal and colonic. This causes severe, long-term constipation or abdominal pain, and can develop secondary to systemic diseases such as Parkinson's Disease or hypothyroidism, although most cases are idiopathic.

Acute colonic pseudo-obstruction (ACPO) described by the authors - also known as Ogilvie's Syndrome [8] — is a clinical entity characterised by severe colonic dilatation with no evidence of underlying mechanical or anatomical cause. The usual symptoms are acute massive abdominal distension with or without associated abdominal pain, nausea or vomiting, and constipation which is not consistently present [7].

Plain abdominal radiographs will reveal a diffusely dilated colon $(\geq 6 \mathrm{~cm})$, but CT is the imaging modality of choice in order to rule out mechanical obstruction. This also allows the confirmation of potential metastasis and differentiation from toxic megacolon (a complication of a variety of infectious, ischaemic or inflammatory diseases of the colon), which is characterised by its hallmark feature of marked bowel wall thickening, loss of haustration, segmental wall thinning, and the appearance of multilayers due to alternating densities of oedematous submucosa and hyperaemic mucosa (target sign) $[9,10]$.

Increased colonic dilatation up to $130 \mathrm{~mm}$ concerned the authors greatly due to a risk of perforation $[7,9]$. The degree and duration of colonic distension determine the pace and sequence of management options. The authors faced the clinical dilemma of whether to treat the patient with conservative measures or to proceed with medical or endoscopic 
decompression of the dilated colon. The conservative measures should be directed towards eliminating or reducing factors known to contribute to the problem, and usually involve correcting electrolyte abnormalities or fluid resuscitation, ceasing opiates or any antimotility agents, and discontinuing oral intake with or without nasogastric tube decompression $[7,9]$.

Medical decompression with neostigmine is regarded as the initial therapy of choice for patients not responding to conservative therapy, provided if there are no contraindications to its use. This acetylcholinesterase inhibitor causes a large high amplitude of colonic peristalsis and subsequent flatus with bowel movement. It should be noted that the parasympathetic stimulation can cause not only subsequent abdominal discomfort, emesis, and excessive salivation, but also a profound bradycardia, meaning that a monitored cardiac setting is required [7]. In general, if a patient fails to respond after two doses of neostigmine, colonoscopic decompression is advised $[7,11]$.

Successful colonoscopic decompression has been reported in many retrospective series, but its efficacy has not been established in randomised clinical trials. It is usually performed without a preceding oral bowel preparation or enema. Colonoscopy without gas insufflation can be a technically challenging procedure and carries unknown risks [11].

Percutaneous decompression (cecostomy) is invasive and can be complicated by local infection or bleeding [11]. Percutaneous endoscopic colostomy (PEC) provides an alternative management option with the placement of PEC tubes in the caecum by a combined radiological or colonoscopic approach. The major advantage of PEC is the avoidance of general anaesthesia, but the method has not been compared to other methods of decompression $[7,11]$.

The exact pathophysiology of ACPO remains a topic of investigation, and no effective prevention strategy is known $[7,11]$. In 1948, Ogilvie [8] first described ACPO in two patients with retroperitoneal malignancy and hypothesised that the carcinoma was responsible for disrupting the balance between the parasympathetic and sympathetic nervous system, but the specific mechanism of action has not yet been revealed. It is probably impaired a spinal reflex of defecation which is triggered by distention of the rectal wall. The defecation reflex involves the evacuation of faeces from the rectum in response to stimulation of afferent nerves in the distal bowel. The myenteric defecation reflex is responsible for eliciting a contraction of the smooth muscle of the colon and rectum, and propelling the stool toward the rectum. Simultaneous inhibition of somatic efferent pathways to the external anal sphincter permit concurrent opening of the anal canal [7].

The patient described by Nowak et al. [6] had trans-anal decompression. Potential surgery (subtotal resection with primary anastomosis) in the near future had to be taken into account to prevent a colonic perforation. However, the authors' published result of therapy with botulinum neurotoxin (BoNT) raises the question as to whether failure of the anal sphincter to relax plays a crucial role in the physiology of Ogilvie's Syndrome? Should we in fact regard the syndrome as an escalation of an outlet obstruction type of constipation, and consider whether BoNT should be applied earlier, given that the problem with constipation started after the stroke? About 20 years ago, observational studies suggested that the application of BoNT releases the blockage in glyceryl trinitrate bioactivation in smooth muscle cells and suppresses basal continuous sympathetic activity, causing modulation of anal sphincters [12]. That explanation is consistent with the current theory that favours a relative excess of sympathetic over parasympathetic tone of anal sphincters $[13,14]$.

It is impossible to say whether the patient had impaired rectal propulsion with paradoxical contraction of anal sphincters (type II of pelvic dyssynergia) or with incomplete relaxation of anal sphincters (type IV of pelvic dyssynergia) [15]. Contradicting Albanese and Cadeddu (pioneers of BoNT injections for anal dyssynergia), a Polish team applied 125 units of abobotulinumtoxin A for spastic pelvic floor syndrome without transrectal ultrasonography or electromyographic control $[15,16]$. That was a novel approach for BoTN injections over 15 years ago [15]. I am pleased to read that the algorithm for inappropriate anal sphincter contraction found epigones without seeking a diagnostic method for locating the place for injection.

In conclusion, Nowak et al. [6] present a very interesting case of stroke complicated with Ogilvie's Syndrome where for the first time BoNT has been applied as a successful remedy. This may work over 8-12 weeks, which is an advantage over the therapy with neostigmine due to the half-life of neostigmine being only one to two hours [7]. Although the BoNT requires $24-72$ hours to take effect, peaking at about 10 days, its long-lasting effect and good safety profile may challenge other therapies which are not entirely effective approaches in preventing the recurrence of Ogilvie's Syndrome.

\section{References}

1. Li J, Yuan M, Liu Y, et al. Incidence of constipation in stroke patients: A systematic review and meta-analysis. Medicine (Baltimore). 2017; 96(25): e7225, doi: 10.1097/MD.0000000000007225, indexed in Pubmed: 28640117.

2. Sumida K, Molnar MZ, Potukuchi PK, et al. Constipation and risk of death and cardiovascular events. Atherosclerosis. 2019; 281: 114-120.

3. Honkura K, Tomata Y, Sugiyama K, et al. Defecation frequency and cardiovascular disease mortality in Japan: The Ohsaki cohort study. Atherosclerosis. 2016; 246: 251-256, doi: 10.1016/j.atherosclerosis.2016.01.007, indexed in Pubmed: 26812003.

4. Durgan DJ, Lee J, McCullough LD. Bryan, Jr RM. Examining the Role of the Microbiota-Gut-Brain Axis in . Stroke. 2019; 50: 2270-2277.

5. Chen R, Xu Y, Wu P, et al. Transplantation of fecal microbiota rich in short chain fatty acids and butyric acid treat cerebral ischemic stroke by regulating gut microbiota. Pharmacol Res. 2019 Oct. ; 148: 104403.

6. Nowak P, Gala-Błądzińska A, Stybel K, et al. Botulinum toxin in the treatment of intestinal pseudo-obstruction following a stroke. Neurol 
Neurochir Pol. 2020 [Epub ahead of print], doi: 10.5603/PJNNS. a2020.0073, indexed in Pubmed: 33026647.

7. Wells $\mathrm{Cl}, \mathrm{O}$ 'Grady G, Bissett IP. Acute colonic pseudo-obstruction: A systematic review of aetiology and mechanisms. World J Gastroenterol. 2017; 23(30): 5634-5644, doi: 10.3748/wjg.v23.i30.5634, indexed in Pubmed: 28852322.

8. OGILVIE H. Large-intestine colic due to sympathetic deprivation; a new clinical syndrome. Br Med J. 1948; 2(4579): 671-673, doi: 10.1136/ bmj.2.4579.671, indexed in Pubmed: 18886657.

9. Chudzinski AP, Thompson EV, Ayscue JM. Acute colonic pseudoobstruction. Clin Colon Rectal Surg. 2015; 28(2): 112-117, doi: 10.1055/s0035-1549100, indexed in Pubmed: 26034408.

10. Jaffe T, Thompson WM. Large-Bowel Obstruction in the Adult: Classic Radiographic and CT Findings, Etiology, and Mimics. Radiology. 2015; 275(3): 651-663, doi: 10.1148/radiol.2015140916, indexed in Pubmed: 25997131.

11. Jain A, Vargas HD. Advances and challenges in the management of acute colonic pseudo-obstruction (ogilvie syndrome). Clin Colon Rectal Surg. 2012; 25(1): 37-45, doi: 10.1055/s-0032-1301758, indexed in Pubmed: 23449274.
12. Madalinski MH, Slawek J, Madalinski MH, et al. Topical nitrates and the higher doses of botulinum toxin for chronic anal fissure. Hepatogastroenterology. 2001; 48(40): 977-979, indexed in Pubmed: 11490852

13. Jones OM, Brading AF, McC Mortensen NJ. Phosphodiesterase inhibitors cause relaxation of the internal anal sphincter in vitro. Dis Colon Rectum. 2002; 45(4): 530-536, doi: 10.1007/s10350-004-6235-8, indexed in Pubmed: 12006938.

14. Madalinski M, Kalinowski L. Novel options for the pharmacological treatment of chronic anal fissure-role of botulin toxin. Curr Clin Pharmacol. 2009; 4(1): 47-52, doi: 10.2174/157488409787236083, indexed in Pubmed: 19149501.

15. Sławek J, Madaliński M. Botulinum Toxin Therapy for Nonmotor Aspects of Parkinson's Disease. Int Rev Neurobiol. 2017; 134: 1111-1142, doi: 10.1016/bs.irn.2017.04.012, indexed in Pubmed: 28805566.

16. Madalinski M, Chelstowska M, Dlugosinska J. New method of botulinum toxin injection for the treatment of spastic pelvic floor syndrome. XI Polish Congress of Gastroenterology. Warszawa, Poland 2004 (p 280. ; 2004: V17. 